\title{
Códigos de Dispersão Linear para Canais MIMO com Realimentação Limitada
}

\author{
Renato Machado e Bartolomeu F. Uchôa Filho
}

\begin{abstract}
Resumo - Neste artigo, propõem-se códigos de dispersão linear (LDCs) para canais de comunicação sem fio com múltiplas antenas transmissoras, múltiplas antenas receptoras (MIMO) e canal de realimentação limitado. O esquema proposto seleciona o LDC, de um conjunto de vários LDCs, que minimiza a probabilidade de erro instantânea baseado nas condições instantâneas do canal. A determinação do melhor conjunto de LDCs, ou seja, aquele que minimiza a probabilidade de erro média, é descrita como um problema de otimização. Enquanto este problema parece ser em geral intratável matematicamente, para certos parâmetros são apresentados bons conjuntos de LDCs, obtidos a partir de um algoritmo de otimização iterativo. Resultados são apresentados somente para LDCs com taxa unitária, todavia, esta restrição pode ser removida. Resultados de simulação mostram que, para um número fixo de bits de realimentação, os esquemas propostos superam em desempenho de erro os esquemas anteriores.
\end{abstract}

Palavras-Chave-Códigos de dispersão linear, códigos espácio-temporais de bloco, diversidade na transmissão, realimentação limitada, seleção de antenas, seleção de códigos, sistemas MIMO, V-BLAST.

Abstract - In this paper, we propose linear dispersion codes (LDCs) for multiple-input-multiple-output (MIMO) channels with a prescribed amount of feedback. The proposed scheme selects the LDC from a set of LDCs that minimizes the instantaneous error probability based on the instantaneous channel conditions. The determination of the best set of LDCs, i.e., the one that minimizes the average error probability, is described as a constrained optimization problem. While this problem appears to be intractable in general, for certain parameters we present good sets of LDCs, obtained from an iterative optimization algorithm. Results are given for rate-one LDCs only, but this restriction can be removed. Computer simulations show that the proposed schemes outperform previous schemes for a fixed number of feedback bits.

Keywords - Antenna selection, code selection, limited feedback, linear dispersion codes, MIMO systems, space-time block codes, transmit diversity, V-BLAST.

\section{INTRODUÇÃO}

Sistemas de comunicação sem fio com múltiplas antenas transmissoras e múltiplas antenas receptoras (MIMO)

Renato Machado e Bartolomeu F. Uchôa Filho estão com o Grupo de Pesquisa em Comunicações - Departamento de Engenharia Elétrica, Universidade Federal de Santa Catarina - 88040900, Florianópolis - SC, BRASIL, E-mails: machado@eel.ufsc.br, uchoa@eel.ufsc.br.

Este trabalho foi financiado em parte pelo Conselho Nacional de Desenvolvimento Científico e Tecnológico (CNPq), sob os números de processo 141005/2004-1, 200504/2006-1, 484391/2006-2 e 302286/2004-7. (multiple-input-multiple-output) são conhecidos por proporcionarem um aumento na capacidade de canal [1], [2]. Códigos de dispersão linear (LDCs) (linear dispersion codes), introduzidos em [3], são bons candidatos para transmissão de dados em sistemas MIMO que requerem alta taxa de transmissão. As palavras-código transmitidas por um LDC são formadas pelas combinações lineares, nas dimensões tempo e espaço, de certas matrizes de dispersão, sendo os símbolos de dados transmitidos os coeficientes dessas combinações. A maioria dos LDCs é projetada para otimizar a informação mútua média, logo eles não garantem um bom desempenho de erro [4]. Porém, recentemente, Wang et al. [5] consideraram o projeto de LDCs com o propósito de minimizar a probabilidade de erro média. Eles chamaram a atenção para o fato de que uma fórmula explícita para a probabilidade de erro não existe para esse problema. Portanto, nenhum método de otimização analítico pode ser considerado. Ao invés disso, eles propuseram um método de otimização baseado em simulação. Os esquemas apresentados em [3]-[5] foram projetados para sistemas MIMO sem canal de realimentação.

O desempenho de sistemas MIMO pode ser consideravelmente melhorado se existir um canal de realimentação, de tal maneira que a informação do estado do canal (CSI) (channel state information), isto é, os coeficientes dos desvanecimentos, passem a ser conhecidos pelo transmissor, viabilizando a aplicação da técnica conhecida por optimal beamforming [6]. Todavia, a existência de um canal de realimentação limitado, permitindo apenas alguns poucos bits de realimentação, é uma suposição muito mais realista. Alguns esquemas que exploram a técnica de seleção na transmissão, que podem ser encontrados na literatura, adotam como critério de seleção a minimização da probabilidade de erro instantânea, ou, equivalentemente, a maximização da relação sinal-ruído (SNR) (signal-to-noise ratio) instantânea.

Gore e Paulraj [7] propuseram um esquema assistido por realimentação muito interessante. Nesse esquema, realiza-se a transmissão dos símbolos de informação com um código espácio-temporal de bloco (STBC) (space-time block code) [8] através do melhor sub-conjunto de antenas transmissoras, selecionadas de um conjunto de $M_{T} \geq 3$ antenas transmissoras, onde o critério de seleção é um que minimiza a probabilidade de erro instantânea, ou, equivalentemente, maximiza a SNR instantânea. Eles mostraram que a ordem de diversidade igual a $M_{T}$ é alcançada, como se todas as antenas transmissoras tivessem sido utilizadas.

Chen et al. [9] apresentaram a solução analítica da probabilidade de erro para o então chamado esquema 
de seleção/combinação de máxima razão na transmissão (TAS/MRC) (transmit antenna selection/maximal-ratio combining scheme). Nesse esquema, uma única antena transmissora é selecionada de um conjunto de $M_{T} \geq 2$ antenas transmissoras. Mais uma vez, obtêm-se diversidade na transmissão com ordem igual a $M_{T}$. Eles mostraram que quando o sistema é provido de um canal de realimentação e somente STBCs ortogonais (OSTBCs) são considerados, o OSTBC que provê a maior SNR é aquele que faz uso de apenas uma única antena transmissora.

Seguindo esta mesma linha de pesquisa, idéias semelhantes foram propostas por Machado e Uchôa-Filho em [10] e refinadas posteriormente em [11], [12]. Eles propuseram um esquema híbrido de seleção antena/código na transmissão que escolhia a partir de uma lista de STBCs (ortogonais e não-ortogonais) o melhor código para ser utilizado com um certo subconjunto de antenas transmissoras, baseado nos valores dos coeficientes instantâneos dos desvanecimentos. Nesta mesma direção, os chamados códigos grupo-coerentes (GCCs) (group-coherent codes) foram propostos por Akhtar e Gesbert [13], [14] para $M_{T}=p M$ antenas transmissoras e $p-1$ bits de realimentação. Em [15], os GCCs foram generalizados, resultando em um melhor desempenho de erro em sistemas com $(p-1) \log _{2}(r)$ bits de realimentação, onde $r$ é um número positivo e potência de 2 .

Para todos os esquemas descritos anteriormente, nenhum conjunto de códigos foi otimizado formalmente, e suas escolhas foram baseadas em intuição. Neste artigo, para um sistema de comunicação sem fio com $M_{T} \geq 2$ antenas transmissoras, $M_{R}$ antenas receptoras e realimentação limitada, propõe-se um esquema de transmissão com seleção de códigos (CS) (code selection) que seleciona o melhor LDC de um conjunto de $2^{b_{f}}$ LDCs, onde $b_{f}$ é o número de bits de realimentação. Por melhor LDC, entende-se o LDC que minimiza a probabilidade de erro instantânea.

Ainda neste artigo, para uma quantidade prescrita de bits de realimentação, a determinação do melhor conjunto de LDCs, ou seja, aquele que minimiza a probabilidade de erro média, é descrita como um problema de otimização. A solução geral desse problema, no entanto, é demasiada complicada. Porém, para certos valores de $M_{T}, b_{f}$ e número de símbolos de informação, obteve-se bons conjuntos de LDCs através do uso de um algoritmo de otimização iterativa. Resultados são apresentados somente para LDCs com taxa de transmissão unitária, mas esta restrição pode ser removida.

Na próxima seção, faz-se uma revisão sobre os LDCs. O esquema proposto e o problema de otimização associado a ele são descritos na Seção III. Na Seção IV, descreve-se o algoritmo de otimização iterativa usado para se obter os bons conjuntos de LDCs para certos parâmetros selecionados, os quais são apresentados na Seção V. Na Seção VI, os resultados de simulação são apresentados. Finalmente, a Seção VII conclui o artigo com alguns comentários finais.

\section{Códigos De Dispersão Linear}

Nesta seção, apresenta-se o modelo do canal para sistemas MIMO.

\section{A. Modelo do Canal}

Neste artigo, considera-se um sistema MIMO com $M_{T}$ antenas transmissoras e $M_{R}$ antenas receptoras. Assumese que o canal sofre desvanecimento do tipo Rayleigh, plano e permanece constante por $\tau$ intervalos de símbolo. A relação entrada-saída deste sistema é dada por:

$$
\mathbf{Y}=\gamma_{0} \mathbf{X H}+\mathbf{W},
$$

onde $\mathbf{Y}$ é a matriz, de dimensões $\tau \times M_{R}$, que contém os sinais recebidos; $\mathbf{X}$ é a matriz, de dimensões $\tau \times M_{T}$, que contém os sinais transmitidos; $\mathbf{W}$ é a matriz, de dimensões $\tau \times M_{R}$, que representa o ruído aditivo Gaussiano circularmente simétrico, com média zero e variância unitária $\mathcal{C N}\left(\mathbf{0}, \mathbf{I}_{\tau M_{R}}\right) ; \mathbf{H}$ é a matriz canal MIMO, de dimensões $M_{T} \times M_{R} ; \gamma_{0}=\sqrt{\frac{\rho}{M_{T}}}$, e $\rho$ é a SNR média em cada antena receptora, indiferentemente do número de antenas receptoras. Considera-se que os símbolos de informação $\left\{s_{q}\right\}, q=1, \ldots, Q$, pertençam a uma constelação complexa de $Q$ sinais $r$-QAM com energia média unitária, e são transmitidos sobre $\tau$ intervalos de símbolo. A matriz de dispersão linear $\mathbf{X}$ é dada por [3]

$$
\mathbf{X}=\sum_{q=1}^{Q} \alpha_{q} \mathbf{A}_{q}+j \beta_{q} \mathbf{B}_{q},
$$

onde os símbolos transmitidos $s_{q}$ são divididos em parte real e parte imaginária, ou seja, $s_{q}=\alpha_{q}+j \beta_{q} .\left\{\mathbf{A}_{q}, \mathbf{B}_{q}\right\}$, $q=1, \ldots, Q$, são matrizes de dispersão complexas, de dimensões $\tau \times M_{T}$, que especificam o código. A taxa de transmissão de um LDC é dada por:

$$
R=\frac{Q \log _{2} r}{\tau} \text { (bit/intervalo de símbolo). }
$$

Tipicamente, as matrizes de dispersão $\left\{\mathbf{A}_{q}, \mathbf{B}_{q}\right\}, q=$ $1, \ldots, Q$, devem satisfazer uma das seguintes restrições de energia, listadas em ordem crescente de restringência [3]:

$$
\begin{gathered}
\sum_{q=1}^{Q} \operatorname{Tr}\left(\mathbf{A}_{q}^{\mathcal{H}} \mathbf{A}_{q}+\mathbf{B}_{q}^{\mathcal{H}} \mathbf{B}_{q}\right)=2 \tau M_{T} ; \\
\operatorname{Tr} \mathbf{A}_{q}^{\mathcal{H}} \mathbf{A}_{q}=\operatorname{Tr} \mathbf{B}_{q}^{\mathcal{H}} \mathbf{B}_{q}=\frac{\tau M_{T}}{Q}, \quad q=1, \ldots, Q ; \\
\mathbf{A}_{q}^{\mathcal{H}} \mathbf{A}_{q}=\mathbf{B}_{q}^{\mathcal{H}} \mathbf{B}_{q}=\frac{\tau}{Q} I_{M_{T}}, \quad q=1, \ldots, Q,
\end{gathered}
$$

onde $(\cdot)^{\mathcal{H}}$ denota a operação conjugado transposto. Definem-se $\mathbf{Y}_{R} \triangleq \Re\{\mathbf{Y}\}$ e $\mathbf{Y}_{I} \triangleq \Im\{\mathbf{Y}\}$. Denota-se a $n$ ésima coluna de $\mathbf{Y}_{R}, \mathbf{Y}_{I}, \mathbf{H}_{R}, \mathbf{H}_{I}, \mathbf{W}_{R}$ e $\mathbf{W}_{I}$, respectivamente, por $\mathbf{y}_{R, n}, \mathbf{y}_{I, n}, \mathbf{h}_{R, n}, \mathbf{h}_{I, n}, \mathbf{w}_{R, n}$ e $\mathbf{w}_{I, n}$, e definemse

$$
\begin{gathered}
\mathcal{A}_{q} \triangleq\left[\begin{array}{cc}
\mathbf{A}_{R, q} & -\mathbf{A}_{I, q} \\
\mathbf{A}_{I, q} & \mathbf{A}_{R, q}
\end{array}\right], \quad \mathcal{B}_{q} \triangleq\left[\begin{array}{cc}
-\mathbf{B}_{I, q} & -\mathbf{B}_{R, q} \\
\mathbf{B}_{R, q} & -\mathbf{B}_{I, q}
\end{array}\right], \\
\underline{\mathbf{h}}_{n} \triangleq\left[\begin{array}{c}
\mathbf{h}_{R, n} \\
\mathbf{h}_{I, n}
\end{array}\right]
\end{gathered}
$$


onde $n=1, \ldots, M_{R}$, e $\mathbf{A}_{R, q}, \mathbf{A}_{I, q}, \mathbf{B}_{R, q}$ e $\mathbf{B}_{I, q}$ são as partes real e imaginária das matrizes $\mathbf{A}_{\mathbf{q}}$ e $\mathbf{B}_{\mathbf{q}}$, respectivamente. Em posse dessas definições, pode-se reescrever (1) da seguinte forma:

$$
\underbrace{\left[\begin{array}{c}
\mathbf{y}_{R, 1} \\
\mathbf{y}_{I, 1} \\
\vdots \\
\mathbf{y}_{R, M_{R}} \\
\mathbf{y}_{I, M_{R}}
\end{array}\right]}_{\mathbf{y}}=\sqrt{\frac{\rho}{M_{T}}} \mathcal{H} \underbrace{\left[\begin{array}{c}
\alpha_{1} \\
\beta_{1} \\
\vdots \\
\alpha_{Q} \\
\beta_{Q}
\end{array}\right]}_{\mathbf{x}}+\underbrace{\left[\begin{array}{c}
\mathbf{w}_{R, 1} \\
\mathbf{w}_{I, 1} \\
\vdots \\
\mathbf{w}_{R, M_{R}} \\
\mathbf{w}_{I, M_{R}}
\end{array}\right]}_{\mathbf{w}},
$$

onde a matriz real de canal equivalente, $\mathcal{H}$, de dimensões $2 M_{R} \tau \times 2 Q$ é dada por

$$
\mathcal{H}=\left[\begin{array}{ccccc}
\mathcal{A}_{1} \underline{\mathbf{h}}_{1} & \mathcal{B}_{1} \underline{\mathbf{h}}_{1} & \ldots & \mathcal{A}_{Q} \underline{\mathbf{h}}_{1} & \mathcal{B}_{Q} \underline{\mathbf{h}}_{1} \\
\vdots & \vdots & \ddots & \vdots & \vdots \\
\mathcal{A}_{1} \underline{\mathbf{h}}_{M_{R}} & \mathcal{B}_{1} \underline{\mathbf{h}}_{M_{R}} & \ldots & \mathcal{A}_{Q} \underline{\mathbf{h}}_{M_{R}} & \mathcal{B}_{Q} \underline{\mathbf{h}}_{M_{R}}
\end{array}\right]
$$

Os LDCs incluem, como casos especiais, ambos V-BLAST [17] e os OSTBCs [8]. Uma importante característica dos LDCs é sua simplicidade de codificação (2). Além disso, os LDCs podem ser decodificados de maneira muito eficiente através de algoritmos bem conhecidos da literatura, tais como: o decodificador esférico [18], o decodificador seqüêncial de Monte-Carlo [19], o detector de anulamento e cancelamento [20], bem como os detectores lineares [21].

\section{Códigos de Dispersão linear para Canais MIMO COM REALIMENTAÇÃo LimitadA}

Nesta seção, propõem-se LDCs para canais MIMO com realimentação limitada. Considera-se o sistema MIMO descrito na Seção II-A com $M_{T} \geq 2$ transmissoras e, por simplicidade, $M_{R}=1$ antena receptora. Assume-se que os coeficientes dos desvanecimentos dos canais sejam perfeitamente estimados pelo receptor, e que exista um canal de realimentação totalmente livre de erros e de atrasos, através do qual $b_{f}$ bits possam ser enviados ao transmissor. No esquema de transmissão aqui proposto, baseado nas condições instantâneas do canal, o receptor (considera-se um receptor linear) determina qual LDC, dentro de um conjunto de $2^{b_{f}}$ LDCs, produz a máxima SNR instantânea (isto é, a mínima probabilidade de erro instantânea). Então, o receptor envia um índice $b_{f}$-bit através do canal de realimentação indicando qual LDC (o que produz a maior SNR) deve ser usado pelo transmissor.

Seja $J=\left\{1, \ldots, 2^{b_{f}}\right\}$ um conjunto de índices. Seja $\mathcal{S}=$ $\{\{\mathbf{A}(i), \mathbf{B}(i)\}: i \in J\}$ o conjunto contendo $2^{b_{f}}$ LDCs. Seja $\mathcal{H}(\{\mathbf{A}(i), \mathbf{B}(i)\}, \mathbf{H})$ a matriz em (9) para o $i$-ésimo LDC em $\mathcal{S}$ para uma dada realização de canal $\mathbf{H}$. A probabilidade de erro instantânea em relação ao par (PEP) (pairwise error probability), isto é, a probabilidade condicionada a $\mathcal{H}$ que um receptor de máxima-verossimilhança (ML) (maximum-likelihood) decida erroneamente em favor de um vetor real $\mathbf{e}$ dado que o vetor de dados real $\mathbf{x}$, definido em (8), foi transmitido, é dada por:

$$
P(\mathbf{x} \rightarrow \mathbf{e} \mid \mathcal{H})=P\left(\left\|\mathbf{y}-\gamma_{0} \mathcal{H} \mathbf{e}\right\|^{2}<\left\|\mathbf{y}-\gamma_{0} \mathcal{H} \mathbf{x}\right\|^{2}\right),
$$

que é bem aproximada por [22]

$$
P(\mathbf{x} \rightarrow \mathbf{e} \mid \mathcal{H}) \leqslant \exp \left(-\|\mathcal{H}(\mathbf{x}-\mathbf{e})\|^{2} \frac{\gamma_{0}^{2} M_{T}}{4}\right),
$$

onde $\|\cdot\|^{2}$ denota a norma de Frobenius. A probabilidade de erro em (10) não depende somente da constelação de sinais adotada, mas também dos pares de vetores formados entre os sinais enviados e os sinais detectados erroneamente, o que torna ainda mais complicada a solução deste problema. Ao invés disso, optou-se por uma forma de representar a probabilidade de erro que dependesse essencialmente das condições instantâneas do canal $\mathbf{H}$ e da descrição do LDC, que é obtida pelas matrizes de dispersão $\mathbf{A}$ e $\mathbf{B}$, e que, entretanto, independe da constelação de símbolos adotada. Em função disso, tornou-se apropriado considerar a PEP condicionada a $\mathcal{H}$ obtida em [3]

$$
P_{e}(\mathcal{H}) \leq \frac{1}{2} \operatorname{det}\left[I+\frac{\gamma_{0}^{2}}{2} \mathcal{H}^{T} \mathcal{H}\right]^{-1 / 2},
$$

onde $(\cdot)^{T}$ denota a operação transposto. Nesta formula, assume-se que as entradas do sistema são do tipo Gaussiana, o que a torna independente de qualquer constelação que seja adotada. Em nosso esquema de transmissão, considerando-se uma dada realização de canal $\mathbf{H}$ e um dado conjunto $\mathcal{S}$ de LDCs, o receptor seleciona o índice $i^{*}$ correspondente ao melhor LDC, de acordo com a seguinte equação:

$$
i^{*}=\arg \min _{i} P_{e}(\mathcal{H}(\{\mathbf{A}(i), \mathbf{B}(i)\}, \mathbf{H})) .
$$

Descreve-se agora o problema de otimização relacionado com a determinação do conjunto ótimo de LDCs. O objetivo aqui é encontrar o conjunto de LDCs que minimize a PEP média sobre todas as realizações de canal. Seja

$$
\bar{P}_{e}(\mathcal{S})=E_{\mathbf{H}} \min _{\{\mathbf{A}, \mathbf{B}\} \in \mathcal{S}} P_{e}(\mathcal{H}(\{\mathbf{A}, \mathbf{B}\}, \mathbf{H}))
$$

a PEP média para o conjunto $\mathcal{S}$ de LDCs. É importante observar que um LDC $\{\mathbf{A}, \mathbf{B}\}$ é um ponto no espaço complexo $\mathbb{C}^{2 \tau M_{T} Q}$. Logo, um conjunto $\mathcal{S}$ é um ponto no espaço complexo $\underbrace{\mathbb{C}^{2 \tau M_{T} Q} \times \cdots \times \mathbb{C}^{2 \tau M_{T} Q}}_{2^{2_{f}} \text { times }}$. Portanto, o conjunto ótimo $\mathcal{S}^{*}$ de LDCs (sujeito a uma das restrições de energia em Sec. II-A) é dado por

$$
\mathcal{S}^{*}=\arg \underbrace{\mathbb{C}^{2 \tau M_{T} Q} \times \cdots \times \mathbb{C}^{2 \tau M_{T} Q}}_{\text {s }^{b_{f}} \text { times }} \bar{P}_{e}(\mathcal{S}) .
$$

Conforme foi observado em [5], a minimização do valor esperado de (12) não pode ser resolvida analiticamente. O problema de otimização em (15), que é muito mais complexo, deve, portanto, ser resolvido através de algum método baseado em simulação. Nesse caso, o espaço complexo multidimensional no qual as matrizes de dispersão linear residem, deve estar restrito a algum sub-espaço finito, e esperanças em relação a $\mathbf{H}$ devem ser obtidas através de validações sobre um grande número de realizações de canal. 


\section{OtimizaÇÃo Iterativa}

Nesta seção, descreve-se o algoritmo de otimização iterativa usado para encontrar os conjuntos de códigos que são apresentados na Seção V. Devido às restrições de espaço, o algoritmo iterativo é descrito somente para os parâmetros: $M_{T}=2, M_{R}=1, \tau=Q=1$ e $b_{f}=1$. Considera-se a função custo em (15). A restrição de energia em (5) é adotada.

Em nosso exemplo, cada iteração é dividida em quatro sub-iterações. As matrizes de dispersão $\mathbf{A}_{1}^{(m, n)}(i)$ e $\mathbf{B}_{1}^{(m, n)}(i)$, para $i=1,2$, produzidas no final de cada subiteração $n$ da iteração $m$, são definidas como:

$$
\begin{aligned}
& \mathbf{A}_{1}^{(m, n)}(1) \triangleq\left[\begin{array}{l}
a_{4}^{(m, n)} \\
a_{3}^{(m, n)}
\end{array}\right]^{T} \quad \mathbf{B}_{1}^{(m, n)}(1) \triangleq\left[\begin{array}{c}
b_{4}^{(m, n)} \\
b_{3}^{(m, n)}
\end{array}\right]^{T} \\
& \mathbf{A}_{1}^{(m, n)}(2) \triangleq\left[\begin{array}{l}
a_{2}^{(m, n)} \\
a_{1}^{(m, n)}
\end{array}\right]^{T} \quad \mathbf{B}_{1}^{(m, n)}(2) \triangleq\left[\begin{array}{l}
b_{2}^{(m, n)} \\
b_{1}^{(m, n)}
\end{array}\right]^{T}
\end{aligned}
$$

O algoritmo é inicializado com $a_{k}^{(0,4)}=b_{k}^{(0,4)}=1$, $k=2,3,4$. No começo da sub-iteração $n$ da iteração $m$, as matrizes auxiliares $\tilde{\mathbf{A}}(i)$ e $\tilde{\mathbf{B}}(i)$, para $i=1,2$, são definidas como:

$$
\begin{aligned}
& \tilde{\mathbf{A}}(1) \triangleq\left[\begin{array}{c}
\tilde{a}_{4} \\
\tilde{a}_{3}
\end{array}\right]^{T} \quad \tilde{\mathbf{B}}(1) \triangleq\left[\begin{array}{c}
\tilde{b}_{4} \\
\tilde{b}_{3}
\end{array}\right]^{T} \\
& \tilde{\mathbf{A}}(2) \triangleq\left[\begin{array}{c}
\tilde{a}_{2} \\
\tilde{a}_{1}
\end{array}\right]^{T} \quad \tilde{\mathbf{B}}(2) \triangleq\left[\begin{array}{c}
\tilde{b}_{2} \\
\tilde{b}_{1}
\end{array}\right]^{T}
\end{aligned}
$$

e são formadas da seguinte forma:

e

$$
\tilde{a}_{k}= \begin{cases}\gamma_{1} e^{j \theta_{1}}, & \text { for } k=n \\ a_{k}^{(m, n-1)}, & \text { for } k<n \\ a_{k}^{(m-1,4)}, & \text { for } k>n\end{cases}
$$

$$
\tilde{b}_{k}= \begin{cases}\gamma_{2} e^{j \theta_{2}}, & \text { for } k=n \\ b_{k}^{(m, n-1)}, & \text { for } k<n \\ b_{k}^{(m-1,4)}, & \text { for } k>n\end{cases}
$$

Como uma consequiência da restrição de energia em (5), $\gamma_{1}$ e $\gamma_{2}$ são forçados a ser unitários e, por isso, podem ser omitidos. Essas matrizes são otimizadas em relação às variáveis $\theta_{1}$ e $\theta_{2}$, isto é, a minimização em (15) é resolvida. Neste trabalho, para reduzir o esforço computacional, o par $\left(\theta_{1}, \theta_{2}\right)$ foi restrito ao conjunto $I_{\theta} \times I_{\theta}$, onde $I_{\theta}=$ $\{0, \pi / 10,2 \pi / 10, \ldots, 19 \pi / 10\}$. A esperança em relação a $\mathbf{H}$ em (14) foi obtida através de 20.000 realizações de canais. A sub-iteração $n$ da iteração $m$ termina atribuindose para as matrizes $\mathbf{A}_{1}^{(m, n)}(1), \mathbf{A}_{1}^{(m, n)}(2), \mathbf{B}_{1}^{(m, n)}(1)$ e $\mathbf{B}_{1}^{(m, n)}(2)$ os melhores valores de $\tilde{\mathbf{A}}(1), \tilde{\mathbf{A}}(2), \tilde{\mathbf{B}}(1)$ e $\tilde{\mathbf{B}}(2)$, respectivamente.

Para este algoritmo iterativo, a função custo é reduzida a cada passo e possui um limitante inferior. Logo, o algoritmo deve convergir. O programa realiza iterações até que a função custo alcance um valor estável, que será, no pior caso, um mínimo local.

$\mathrm{O}$ algoritmo iterativo que foi descrito para um exemplo em particular nesta seção pode facilmente ser estendido para outros valores de parâmetros. Bons conjuntos de LDCs são apresentados na próxima seção.

\section{Bons Conjuntos de LDCs para ParÂmetros SELeCionados}

Os resultados obtidos através do algoritmo iterativo descrito na Seção IV são apresentados nesta seção. No final da seção, algumas extensões destes resultados são também apresentadas.

As matrizes de dispersão para os parâmetros $M_{T}=2$, $M_{R}=1, \tau=Q=1$ e $b_{f}=1$ são dadas por:

$$
\mathbf{A}_{1}(1)=\mathbf{B}_{1}(1)=\left[\begin{array}{l}
1 \\
1
\end{array}\right]^{T}, \quad \mathbf{A}_{1}(2)=\mathbf{B}_{1}(2)=\left[\begin{array}{c}
1 \\
-1
\end{array}\right]^{T}
$$

e os códigos correspondentes são dados por:

$$
\mathbf{X}_{1}=\left[\begin{array}{ll}
s_{1} & s_{1}
\end{array}\right], \quad \mathbf{X}_{2}=\left[\begin{array}{ll}
s_{1} & -s_{1}
\end{array}\right] .
$$

Para $M_{T}=2, M_{R}=1, \tau=Q=1$ e $b_{f}=2$, usouse o mesmo algoritmo iterativo descrito anteriormente (adaptado para esta nova configuração) para encontrar o "melhor" conjunto de códigos. O melhor conjunto de LDCs (sujeito à restrição de energia em (5)) foi encontrado. ${ }^{1}$

As matrizes de dispersão para os parâmetros $M_{T}=2$, $M_{R}=1, \tau=Q=1$ e $b_{f}=2$ são dadas por:

$$
\begin{aligned}
& \mathbf{A}_{1}(1)=\mathbf{B}_{1}(1)=\left[\begin{array}{l}
1 \\
1
\end{array}\right]^{T}, \quad \mathbf{A}_{1}(2)=\mathbf{B}_{1}(2)=\left[\begin{array}{l}
1 \\
j
\end{array}\right]^{T}, \\
& \mathbf{A}_{1}(3)=\mathbf{B}_{1}(3)=\left[\begin{array}{c}
1 \\
-1
\end{array}\right]^{T}, \mathbf{A}_{1}(4)=\mathbf{B}_{1}(4)=\left[\begin{array}{c}
1 \\
-j
\end{array}\right]^{T},
\end{aligned}
$$

e os correspondentes códigos são dados por:

$$
\begin{array}{ll}
\mathbf{X}_{1}=\left[\begin{array}{ll}
s_{1} & s_{1}
\end{array}\right], & \mathbf{X}_{2}=\left[\begin{array}{ll}
s_{1} & j s_{1}
\end{array}\right], \\
\mathbf{X}_{3}=\left[\begin{array}{ll}
s_{1} & -s_{1}
\end{array}\right], & \mathbf{X}_{4}=\left[\begin{array}{ll}
s_{1} & -j s_{1}
\end{array}\right] .
\end{array}
$$

Nota-se que a melhor solução para esses simples exemplos são, na realidade, casos especiais dos chamados códigos grupo-coerente generalizados [15]. Para outros valores de parâmetros, a melhor solução é desconhecida. Porém, deve-se notar que, utilizando-se o conjunto de LDCs descrito em (16), o transmissor envia o sinal $s_{1}$ através da antena 1 , e $s_{1}$, ou uma versão rotacionada em $\pi$ radianos de $s_{1}$, através da antena 2 , dependendo de qual seja a diferença de fase existente entre $h_{1,1}$ e $h_{2,1}$. Em outras palavras, o bit de realimentação traz consigo somente informação de fase. Esta mesma idéia pode ser estendida para outros valores de parâmetros, por exemplo, quando o número de LDCs em $\mathcal{S}$ (dado por $2^{b_{f}}$ ) está casado (num sentido que se tornará mais claro) com o número de antenas transmissoras. Embora não tenham sido obtidos através da solução do problema de otimização em (15), os conjuntos de LDCs de taxa unitária (isto é, $\tau=Q$ ) apresentados a seguir são, provavelmente, ótimos também.

\footnotetext{
${ }^{1}$ Deve-se notar que soluções equivalentes são possíveis. Qualquer versão rotacionada dessas soluções também podem ser consideradas soluções "ótimas".
} 
As matrizes de dispersão para os parâmetros $M_{T}=2$, $M_{R}=1, \tau=Q=1$ e $b_{f}=b$, são dadas por:

$$
\mathbf{A}_{k, 1}=\mathbf{B}_{k, 1}=\left[\begin{array}{c}
1 \\
\exp \left(j \frac{2 \pi}{2^{b}}(k-1)\right)
\end{array}\right]^{T},
$$

e os correspondentes códigos são dados por:

$$
\mathbf{X}_{k}=\left[\begin{array}{ll}
s_{1} & \exp \left(j \frac{2 \pi}{2^{b}}(k-1)\right) s_{1}
\end{array}\right],
$$

onde $k=1, \ldots, 2^{b}$.

Estendendo-se para os parâmetros $M_{T}=2, b_{f}=b \mathrm{e}$ $\tau=Q \geq 1$, para $q=1, \ldots, Q$, têm-se que $\mathbf{A}_{k, q}$ e $\mathbf{B}_{k, q}$ são dadas por:

$$
\mathbf{A}_{k, q}=\mathbf{B}_{k, q}=\left[\begin{array}{ccccc}
0 & \ldots & 1 & \ldots & 0 \\
0 & \ldots & \exp \left(j \frac{2 \pi}{2^{b}}(k-1)\right) & \ldots & 0
\end{array}\right]^{T},
$$

onde somente a $q$-ésima coluna é não-nula, e os correspondentes códigos são dados por:

$$
\mathbf{X}_{k}=\left[\begin{array}{cc}
s_{1} & \exp \left(j \frac{2 \pi}{2^{b}}(k-1)\right) s_{1} \\
\vdots & \vdots \\
s_{Q} & \exp \left(j \frac{2 \pi}{2^{b}}(k-1)\right) s_{Q}
\end{array}\right],
$$

onde $k=1, \ldots, 2^{b}$.

Para $M_{T}=3, b_{f}=2 b$ e $\tau=Q \geq 1$, têm-se que $\mathbf{A}_{k, q}$ e $\mathbf{B}_{k, q}$, para $q=1, \ldots, Q$, são da forma:

$$
\mathbf{A}_{k, q}=\mathbf{B}_{k, q}=\left[\begin{array}{ccccc}
0 & \ldots & 1 & \ldots & 0 \\
0 & \ldots & \alpha_{1} & \ldots & 0 \\
0 & \ldots & \alpha_{2} & \ldots & 0
\end{array}\right]^{T}
$$

onde somente a q-ésima coluna é não-nula, e os correspondentes códigos são da forma:

$$
\mathbf{X}_{k}=\left[\begin{array}{ccc}
s_{1} & \alpha_{1} s_{1} & \alpha_{2} s_{1} \\
\vdots & \vdots & \vdots \\
s_{Q} & \alpha_{1} s_{Q} & \alpha_{2} s_{Q}
\end{array}\right]
$$

onde para cada $\mathbf{X}_{k}, k=1, \ldots, 2^{2 b}$, um par $\left(\alpha_{1}, \alpha_{2}\right)$ diferente é tomado de

$$
\left\{\exp \left(j \frac{2 \pi}{2^{b}} n_{1}\right), \exp \left(j \frac{2 \pi}{2^{b}} n_{2}\right)\right\}
$$

onde $n_{1}, n_{2}=0,1, \ldots, 2^{b}-1$.

Uma característica muito importante em relação aos códigos que foram apresentados nesta seção deve ser mencionada. Embora tenha sido considerada nesse problema de otimização uma classe de códigos bastante ampla: a classe dos LDCs, o melhor conjunto de códigos membros desta classe é surpreendentemente simples. Na próxima seção, apresentam-se alguns resultados sobre o desempenho de erro desses novos códigos.

\section{Resultados de Simulação}

Nesta seção, comparam-se os LDCs discutidos na Seção $\mathrm{V}$ em termos de suas taxas de erros de bits (BER) (bit error rate) versus SNR $\left(\gamma_{0}\right)$ para uma modulação BPSK, sujeitos aos efeitos de um desvanecimento do tipo plano, Rayleigh e quasi-estático. Os resultados apresentados na Figura 1 são para o caso de $M_{T}=2$ antenas transmissoras, e na Figura 2, para o caso de $M_{T}=3$ antenas transmissoras, ambas

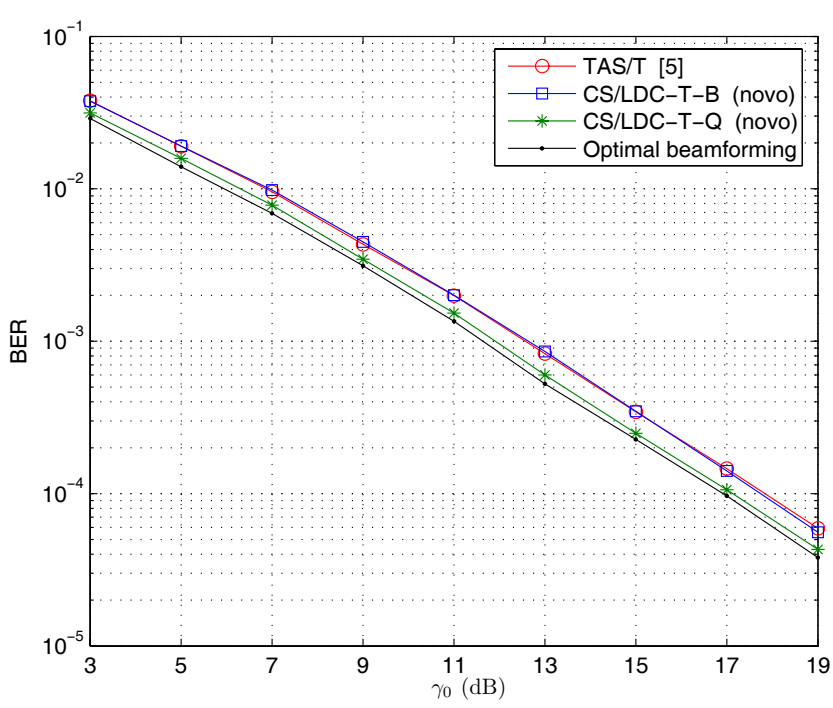

Fig. 1. BER dos esquemas TAS/T $\left(b_{f}=1\right)$, CS/LDC-T-B $\left(b_{f}=1\right)$, CS/LDC-T-Q $\left(b_{f}=2\right)$ e Optimal Beamforming. $M_{T}=2$ e $M_{R}=1$.

para $\tau=Q=1$ e $M_{R}=1$ antena receptora. Em ambas figuras, o esquema conhecido como optimal beamforming é considerado.

Na Figura 1, consideram-se o esquema TAS/T [9] para $b_{f}=1$ bit de realimentação e o esquema CS/LDC-T para $b_{f}=1,2$ bits de realimentação. O esquema CS/LDC$\mathrm{T}$ para $M_{T}=2$ antenas transmissoras é descrito como [s $\alpha_{1} s$ ], onde $\alpha_{1} \in 2^{b_{f}}$-PSK. O grau de diversidade máximo (igual a 2) é alcançado para qualquer número de bits de realimentação.

Na Figura 2, consideram-se os esquemas TAS/A [7], TAS/T [9] e o novo CS/LDC-T-BB, para $b_{f}=2$ bits de realimentação; os esquemas TAS/híbrido-2 [11] e o novo CS/LDC-T-BQ, para $b_{f}=3$ bits de realimentação; e os esquemas HS $3 \mathrm{Tx} 4 \mathrm{Fb}$, apresentado recentemente por Şahin et al. [12], e o novo CS/LDC-T-QQ, para $b_{f}=4$ bits de realimentação.

O esquema CS/LDC-T-BB é descrito em (20) para $b_{f}=2$ bits de realimentação. Em outras palavras, neste esquema, o conjunto de LDCs é definido como um conjunto de quatro matrizes da forma [s $\alpha_{1} s \alpha_{2} s$ ], onde $\alpha_{1}$ e $\alpha_{2} \in$ $\{ \pm 1\}$ (BPSK). O esquema CS/LDC-T-BQ, que não foi descrito anteriormente, corresponde à seleção do melhor código do conjunto de LDCs [ $\left.\begin{array}{lll}s & \alpha_{1} s & \alpha_{2} s\end{array}\right]$, onde $\alpha_{1} \in\{ \pm 1\}$ $\equiv$ BPSK e $\alpha_{2} \in\{ \pm 1, \pm j\} \equiv$ QPSK. O esquema CS/LDCT-QQ é descrito em (20) e corresponde à seleção do melhor código do conjunto de LDCs [ $s \alpha_{1} s \alpha_{2} s$ ], onde $\alpha_{1}$ e $\alpha_{2} \in$ $\{ \pm 1, \pm j\} \equiv$ QPSK. O grau de diversidade máximo (igual a 3) é alcançado para todos esses esquemas.

A partir dos resultados de simulação, pode-se observar que os LDCs propostos neste artigo apresentam o melhor desempenho de erro, para qualquer número de bits de realimentação fixado. Há certas características pertinentes aos conjuntos de códigos de LDCs propostos neste artigo que devem ser mencionadas. Por exemplo, somente uma única cadeia de rádio-frequência $(\mathrm{RF})$ é requerida, pois o mesmo símbolo de informação que é enviado por uma das 


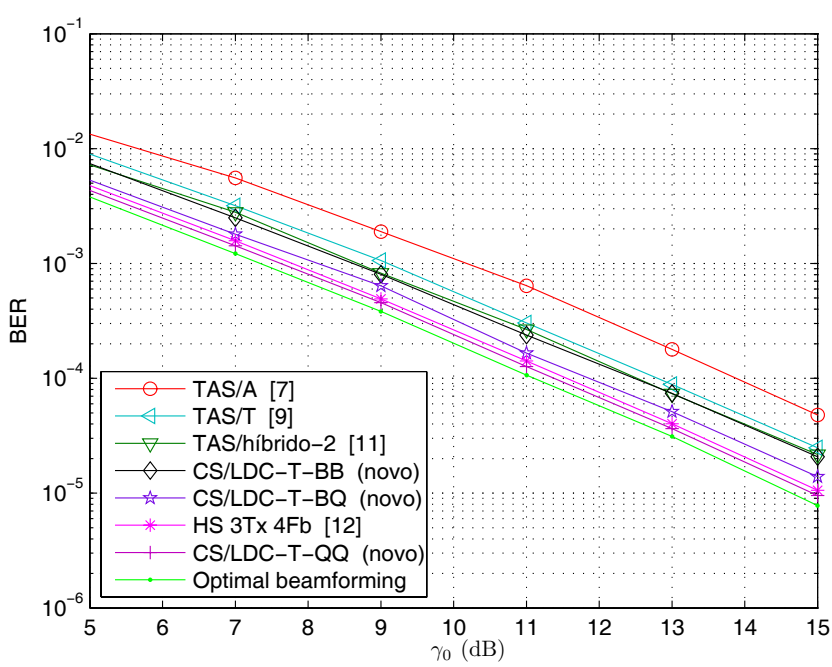

Fig. 2. BER dos esquemas TAS/A, TAS/T e TAS/LDC-T-BB para $b_{f}=2$, TAS/híbrido-2 e CS/LDC-T-BQ para $b_{f}=3$, HS $3 \mathrm{Tx} 4 \mathrm{Fb}$ e TAS/LDC-T-QQ para $b_{f}=4$, e Optimal Beamforming. $M_{T}=3$, $M_{R}=1$.

antenas transmissoras é também enviado por cada uma das outras antenas transmissoras, a não ser por uma possível diferença das rotações nas fases. Além disso, um método de decodificação linear muito simples, com pouco atraso de decodificação ( $\tau=1$ para os LDCs que foram simulados), é adotado.

\section{Conclusões}

Neste artigo, foi proposto um framework geral para seleção de códigos na transmissão com códigos de dispersão linear. Baseado nas condições instantâneas do canal, o transmissor escolhe um LDC de um conjunto de LDCs, objetivando-se minimizar a probabilidade de erro. Nota-se que em todos os conjuntos de LDCs apresentados na Seção $\mathrm{V}$, os bits de realimentação trazem somente informação de fase. Este sempre será o caso quando houver casamento entre os parâmetros, isto é, $\tau=Q$ e $b_{f}=\left(M_{T}-1\right) b$. Outras extensões também são possíveis. Por exemplo, quando $\tau=Q$ e $b_{f}=\sum_{i=1}^{M_{T}-1} b_{i}$, onde $b_{i}$ é um número inteiro positivo, e os sinais transmitidos através das antenas $i+1$ são rotacionados por uma fase de $2^{b_{i}}$-PSK, dependendo da informação que for trazida pelos bits de realimentação. Em todos esses casos, os melhores conjuntos de LDCs, seguindo a mesma idéia apresentada na Seção V, são, provavelmente, os conjuntos ótimos também. No entanto, para os casos em que $b_{f}<M_{T}-1$, a solução ótima não parece ser tão direta e deve envolver uma mistura dos símbolos na transmissão. Nesses casos, para se obter a solução ótima, talvez seja necessário que os bits de realimentação tragam não somente informação de fase, mas também, informação de magnitude.

\section{Agradecimentos}

Os autores agradecem ao Dr. Tolga M. Duman, da Arizona State University (ASU), Arizona, Estados Unidos, por suas sugestões e comentários.

\section{REFERÊNCIAS}

[1] I. E. Telatar, "Capacity of multi-antenna Gaussian channels," Europ. Trans. Telecommun., vol. 10, pp. 585-595, Nov. 1999.

[2] G. J. Foschini, and M. J. Gans, "On limits of wireless communications in a fading environment when using multiple antennas," Wireless Pers. Commun., vol. 6, no. 3, pp. 311-335, Mar. 1998.

[3] B. Hassibi, and B. Hochwald, "High-rate codes that are linear in space and time," IEEE Trans. Inf. Theory, vol. 48, no. 7, pp. 1804-1824, July. 2002.

[4] R. W. Heath. Jr, and A. J. Paulraj, "Linear dispersion codes for MIMO systems based on frame theory," IEEE Trans. Signal Process., vol. 50, no. 10, pp. 2429-2441, Oct. 2002.

[5] X. Wang, V. Krishnamurthy, and J. Wang, "Stochastic gradient algorithms for design of minimum error-rate linear dispersion codes in MIMO wireless systems," IEEE Trans. Signal Process., vol. 54, no. 4, pp. 1242-1255, April 2006.

[6] D. Gore and A. J. Paulraj, "Space-time block coding with optimal antenna selection," in Proc. ICASSP, pp. 2441-2444, May. 2001.

[7] D. Gore and A. Paulraj, "MIMO antenna subset selection with space-time coding," IEEE Trans. on Signal Proc., vol. 50, no. 10, pp. 2580-2588, Dec. 2002.

[8] V. Tarokh, N. Seshadri, and A. R. Calderbank, "Space-time block codes from orthogonal designs," IEEE Trans. Inf. Theory, vol. 45, no. 5, pp. 1456-1467, July. 1999.

[9] Z. Chen, J. Yuan, and B. Vucetic, "Analysis of transmit antenna selection/maximal-ratio combining in Rayleigh fading channels," IEEE Trans. on Vehicular Tecnol. vol. 54, no. 4, pp. 1312-1321, July. 2005.

[10] R. Machado, and B. F. Uchôa-Filho, "Space-time block coding with hybrid transmit antenna/code selection," IEEE Int. Conf. on Commun. (ICC'04), in CR-ROM, June 2004.

[11] R. Machado, and B. F. Uchôa-Filho, "Extended Techniques for Transmit Antenna Selection With STBCs," Journal of Communication and Information Systems (JCIS), submitted for publication in 2006.

[12] S. Şahin, M. E. Çelebi and Ü. Aygölü, "Space diversity schemes with feedback for three transmit antennas," AEU-International Journal of Electronics and Communications, Dec. 2005.

[13] J. Akhtar, and D. Gesbert, "Partial feedback based orthogonal block coding," in Proc. of the 2003 IEEE Vehicular Technology Conf. (VTC'03-Spring), vol. 1, pp. 287-291, April. 2003.

[14] J. Akhtar, and D. Gesbert, "Extending orthogonal block codes with partial feedback," IEEE Trans. on Wireless Commun., vol. 3, pp. 1959-1962, Nov. 2004.

[15] R. Machado, R. dos Santos, and B. F. Uchôa-Filho, "Sobre os Códigos Grupo Coerentes," XXII Simpósio Brasileiro de Telecomunicações (SBrT'05), in CD-ROM, Sept. 2005.

[16] D. Deng, M. Zhao, and J. Zhu, "Transmit Antenna Selection for Linear Dispersion Codes Based on Linear Receiver," in Proc. of the 2006 IEEE Vehicular Technology Conference (VTC'06Spring), vol. 6, pp. 2927-2931, May. 2006.

[17] G. J. Foschini, "Layered space-time architecture for wireless communication in a fading environment when using multiple antennas," Bell Labs. Tech, J., vol. 1, no. 2, pp. 41-59, Autumn. 1996.

[18] M. O. Damen, A. Chkeif, and J. C. Belfiore, "Lattice code decoder for space-time codes," IEEE Commun. Lett., vol. 4, pp. 166-169, May 2000.

[19] B. Dong, X. Wang, and A. Doucet, "A new class of soft MIMO demodulation algorithms," IEEE Trans. Signal Process., vol. 51, no. 11, pp. 2752-2763, Nov. 2003.

[20] G. J. Foschini, G. Golden, R. Valenzuela, and O. Wolniansky, "Simplified processing for high spectral efficiency wireless communication employing multi-element arrays," IEEE J. Sel. Areas Commun., vol. 17, no. 11, pp. 1841-1852, Nov. 1999.

[21] D. Gesbert "Minimum-error linear receivers for ill-conditioned MIMO channels," in Proc. IEEE Workshop Signal Processing Advances in Wireless Communications (SPAWC), pp. 462-466, June. 2003.

[22] V. Tarokh, N. Seshadri, and A. R. Calderbank, "Space-time codes for high data rate communication: performance criterion and code construction," IEEE Trans. on Inform. Theory, vol. 44, no. 2, pp. 744-765, Mar. 1998. 